\title{
Mechanisms of Ion Detection for FET-Sensors Using FTO: Role of Cleaning Process, pH Sequence and Electrical Resistivity
}

\author{
Raphael Aparecido Sanches Nascimento ${ }^{a *}$, Marcelo Mulato ${ }^{b}$ \\ ${ }^{a}$ Department of Physics, Universidade Federal de Lavras, 37200-000, Lavras, MG, Brazil \\ ${ }^{b}$ Department of Physics, Faculty of Philosophy, Sciences and Letters at Ribeirão Preto, Universidade de \\ São Paulo, Av. Bandeirantes 3900, 14040-901, Ribeirão Preto, SP, Brazil
}

Received: May 18, 2017; Revised: June 26, 2017; Accepted: July 03, 2017

\begin{abstract}
The use of FTO samples as an extended gate field effect transistor biosensor is presented. The FTO samples were produced by spray pyrolysis technique. The cleaning process is shown to have a fundamental importance for the final sensitivity of the samples when multiple re-usage is adopted. The role of electrical resistivity and morphology of the films are investigated. The influence of $\mathrm{pH}$ sequence of measurements from 2 to 12 is presented. Both increasing and decreasing the $\mathrm{pH}$ values sequence of measurements are compared. Electrical, morphological, time evolution and electrochemical experiments are correlated in the main discussion. A physical-chemical model is presented to explain the main mechanisms of charge adsorption and desorption. Parameters not commonly reported in the literature are proven to have fundamental importance in sensors behavior and characterization.
\end{abstract}

Keywords: Biosensor, FTO, pH sensor, EGFET

\section{Introduction}

Nanotechnology has provided a true revolution towards miniaturization. In material science and engineering, increasing efforts have been applied to the development of new materials or devices at micro and nanoscale. Materials and devices that have the possibility to be miniaturized to those scales in the future are also studied. This technological appeal is used with quite a success in sensing field.

Among the huge variety of sensors, the electrochemical ones, specially the Extended Gate Field Effect Transistor (EGFET) type of sensors, can be highlighted as really promising devices because of their intrinsic properties such as possible miniaturization, high entrance impedance, low cost, simple equipment required and possibility to use a broad variety of materials including biocompatible ones ${ }^{1}$.Those sensors can be used on the composition of biosensors. Oxides such as $\mathrm{TiO}_{2}^{2}, \mathrm{SnO}_{2}^{3}$ and $\mathrm{ZnO}^{1}$ can be used as sensing part in these devices. The biocompatibility of these materials has already been demonstrated by other research groups ${ }^{4-8}$, and they have been used as part of various types of biosensors.

Interesting works have described the use of EGFET as component of a biosensor to detect, for example, $\mathrm{DNA}^{9,10}$, $\mathrm{CO}_{2}{ }^{11}, \mathrm{O}_{2}{ }^{11}$, Urea ${ }^{12,13}$, Protein ${ }^{14}$, Uric Acid ${ }^{15,16}$ and Glucose ${ }^{17}$. Therefore, the majority of works using EGFET as part of a sensor describes it as a $\mathrm{pH}$-sensor ${ }^{3,18-33}$.

$\mathrm{PH}$ monitoring and control, for itself, are quite important. The human body needs to keep blood's $\mathrm{pH}$ on a very narrow window of value in order to keep the homeostasis. In addition to that, reactions of other important biomolecules as urea $\mathrm{a}^{34}$ and glucose $\mathrm{e}^{35}$ can be indirectly detected through $\mathrm{pH}$ monitoring.
Fluorine Tin Oxide (FTO) is one of the most useful oxide materials. The advantages of using this material rely on its physical/chemical characteristics. For example: FTO is a n-type semiconductor with a large band gap (between $3.9 \sim 4.6 \mathrm{eV})^{36-38}$; when produced by spray pyrolysis method, FTO presents interesting, very promising and useful physicalchemistry characteristics such as potential to be miniaturized, high transparence on visible spectrum region, high surface area, large conductivity range, mechanical hardness, thermal stability and biocompatibility.

Although thin films EGFET biosensors are claimed to work, even with FTO as $\mathrm{pH}$ sensor ${ }^{3}$, specific details during the preparation of each one of them are very important and, very often, seldom reported. For instance, to make these devices reusable, the cleaning process between consecutive measurements must be addressed. At the present work we intend to demonstrate that cleaning process constitutes a very important step describing how the biosensor device works. The cleaning process establishes a starting point for the devices, which can be different for the reuse on further measurements. The role of oxides porous surface in ions adsorption from bulk solutions and the possible masking of the biosensor response will be discussed. This work discusses the importance of doing and describing a careful cleaning process according to the mechanisms involved in the process.

Despite the trivial or simple aspect of the cleaning analysis presented in this work, as seem, they play an important whole in the final sensor response. Moreover, as far as these authors are concerned, most of the previous published work did not presented a significant description of their sensors cleaning process. 


\section{Materials and Methods}

FTO samples produced by Flexitec Corporation were used. They were deposited on glass by spray pyrolysis technique. This technique makes possible a good control of the sheet resistance of the films besides a production of a crystalline material with heterogeneous grain sizes distribution ${ }^{37,38}$. Thin film samples of different nominal sheet resistance of $5 \sim 10$ $\Omega /$ sq (named S10), $21 \Omega /$ sq (named S21) and 80 100 $\Omega /$ sq (named S100) were used in the experiments. Those resistances were also measured in the laboratory by a four-probe system and the values were confirmed with a maximum variance of $\pm 3 \Omega / \mathrm{sq}$. All the samples had the same surface dimensions of $10 \mathrm{~mm}$ width and $26 \mathrm{~mm}$ length. Electric contact was made with a thin cupper wire and conductive silver glue. The encapsulation of this contact was made by an epoxy resin. This encapsulation always took the same area of $3 \mathrm{~mm}$ width and $10 \mathrm{~mm}$ length.

To build the EGFET we used an N-channel of the commercial CD4007 MOSFET from Texas Instruments. Data were acquired using a Data Aquisition HP 34970, and an Agilent E3646A Dual Output DC Power Supply. A homemade software, wrote in LabView 8.0, was used to control the power supply and record the data. Phosphate/ Citrate $(0.1 \mathrm{M})$ homemade buffer solutions were used for pHs 2 to 8 . Solutions with pHs 10 and 12 were made by water titration with $1 \mathrm{M} \mathrm{NaOH}$ solution (purchased from PANREAC).

Figure 1(a) shows a scheme of an ion sensor based on EGFET. The sensing part is totally submerged in the electrolyte solution. The film is linked by wire to the gate of the N-channel MOSFET. Hence, the higher the gate potential, the larger the current flowing through drain towards source in the MOSFET. The reference electrode is also inserted into solution. This electrode is externally biased through a reference electrode setting the bulk of solution to a constant potential $\mathrm{V}_{\mathrm{Ref}}$, see Figure 1-a. Ions from the solution will eventually attach to the surface of the sensor due to its affinity with these ions. Their amount and specificity will change according to each solution and sensing film. The net result will lead to an extra variable potential $\Delta \mathrm{V}$ between bulk solution and the surface of the film. Thus, the final potential difference between gate and source of the transistor will be $\mathrm{V}_{\mathrm{GS}}=\mathrm{V}_{\mathrm{Ref}}+\Delta \mathrm{V}$ (Figure 1 (a)). $\Delta \mathrm{V}$ is threshold voltage as already explained somewhere else ${ }^{39,40}$.

Basically there are two ions being constantly adsorbed or desorbed from the surface: hydrogen $\left(\mathrm{H}^{+}\right)$and hydroxyl $\left(\mathrm{OH}^{-}\right) . \mathrm{H}^{+}$is a very tiny ion (the tiniest possible ion) that can move through the electrolyte and penetrate the surface of the sample very easily. $\mathrm{OH}^{-}$, on the other hand, is a much larger molecule that cannot move around the electrolyte as easily.

Hydrogen ions raise the electrostatic potential of the sample when attached to its surface. On the other hand, hydroxyl molecules lower the electrostatic potential of the sample. This is schematically shown in Figure 1(b). At the

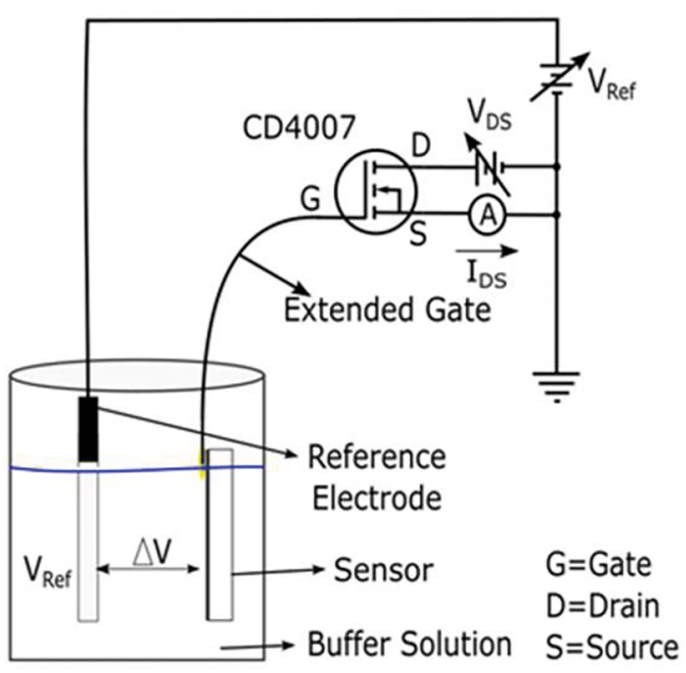

a)

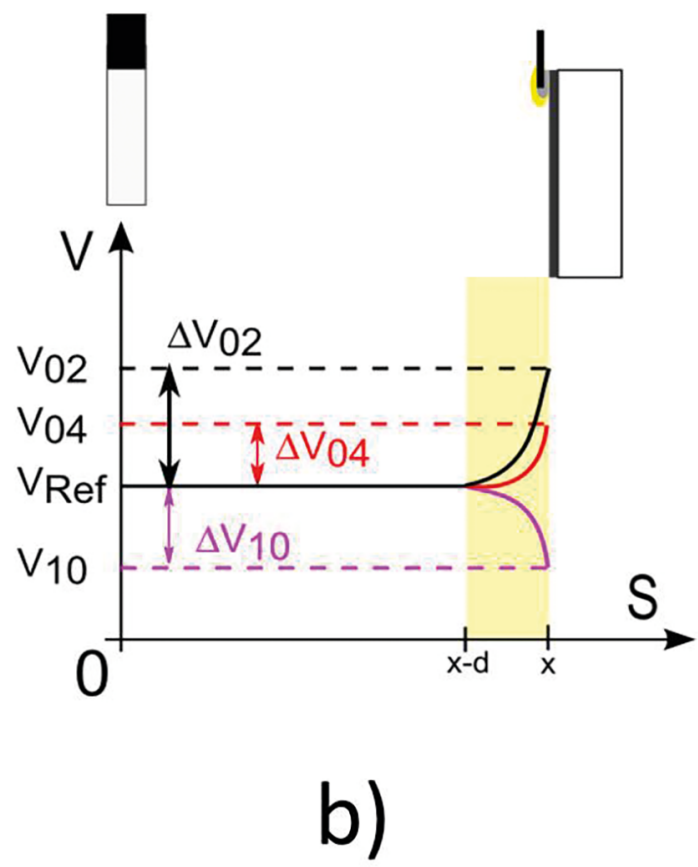

Figure 1. a) The whole apparatus to use an EGFET as a biosensor. b) Electrostatic potential distribution though the space. The reference electrode sits at the origin. The film sits at the $\mathrm{x}$-position. The double layer extends a distance $\mathrm{d}$ from the surface of the sample. $\Delta \mathrm{V}_{02}$ is the voltage variation between reference electrode and sample surface (in double layer region) due to the action of $\mathrm{pH} 02 ; \Delta \mathrm{V}_{04}$ is the voltage variation between reference electrode and sample surface (in double layer region) due to the action of $\mathrm{pH} 04 ; \Delta \mathrm{V}_{10}$ is the voltage variation between reference electrode and sample surface (in double layer region) due to the action of $\mathrm{pH} 10$.

illustration, a graphic composed by Potential (vertical axis) vs Space (horizontal axis) is presented. The reference electrode is placed in the electrolyte bulk solution at the $\mathrm{x}$-axis origin. 
The surface of the sample sits at an $\mathrm{X}$ distance away from the reference electrode. The double layer extends a distance $\mathrm{d}$ from the sample towards the reference electrode. It is exactly within the double layer that the potential is changed by the ions adsorbed on the surface. The electrostatic potential inside the electrolyte solution and out of the double layer area is practically constant and equal to the same potential applied by the reference electrode $\left(\mathrm{V}_{\mathrm{Ref}}\right)$. Depending on which ion is mostly adsorbed on the surface of the sample and also on its concentration, the potential is gradually changed at the double layer area by an amount equal to $\Delta \mathrm{V}$. For example, $\Delta \mathrm{V}_{02}$ is the voltage variation between reference electrode and sample surface (in double layer region) due to the accumulation of proton ions of $\mathrm{pH} 02$ (see Figure 1 (b)). The final $\mathrm{V}_{\mathrm{GS}}$ will be the sum of $\mathrm{V}_{\mathrm{Ref}}+\Delta \mathrm{V}$. The final $\mathrm{V}_{\mathrm{GS}}$ potential will control the size of the channel inside the transistor, and consequently, the current between drain and source of the transistor $\left(\mathrm{I}_{\mathrm{DS}}\right)$. In other words, the current $\mathrm{I}_{\mathrm{DS}}$ will be controlled by the number of ions attached to the sensor.

In the scheme of Figure 1(a), two variable power sources are used, named $\mathrm{V}_{D S}$ and $\mathrm{V}_{\text {Ref }}$, respectively. The current $\mathrm{I}_{\mathrm{DS}}$ is changed when either one of the two power sources is varied. This leads to two different measurement configurations to record the data, here called $\mathrm{V}_{\mathrm{DS}}$ and $\mathrm{V}_{\mathrm{GS}}$. In the $\mathrm{V}_{\mathrm{DS}}$ configuration, $\mathrm{V}_{\text {Ref }}$ is kept constant and $\mathrm{V}_{\mathrm{DS}}$ is varied from 0 to 5 volts, in $0.1 \mathrm{~V} / \mathrm{s}$ steps. The name $\mathrm{V}_{\mathrm{DS}}$ is due to the selected voltage source to change along the time. In this case, the voltage source selected is $\mathrm{V}_{\mathrm{DS}}$ (the voltage between drain and source for the MOSFET - see Figure 1 (a)). The current $I_{D S}$ is the current that passes by the Drain (D) and comes out of the Source (S) terminal of the MOSFET towards the circuit Ground - see Figure 1 (a)). On the other hand, in the $\mathrm{V}_{\mathrm{GS}}$ configuration, $\mathrm{V}_{\mathrm{DS}}$ is kept constant and $\mathrm{V}_{\text {Ref }}$ is varied from 0 to 5 volts, also in $0.1 \mathrm{~V} / \mathrm{s}$ steps. The $\mathrm{V}_{\mathrm{GS}}$ mode is used to obtain the sensitivity of the samples ${ }^{41}$. The name, $\mathrm{V}_{\mathrm{GS}}$, is due to the voltage source that changes is the source named as $\mathrm{V}_{\text {Ref }}$, and consequently varying $\mathrm{V}_{\mathrm{GS}}$ (between source and gate of the MOSFET - see Figure 1 (a)).

Different procedures were used to clean the samples, using two different solutions. For the first one the sample was cleaned with DI water before each measurement. The samples prepared according to this procedure will be named sample "Wt" which stands for water. This cleaning consists on just squeezing water on the top of the sample by approximately five seconds, followed by tissue paper drying. The second procedure starts with the same recipe as the previous one, followed by resting the sample inside an alkaline solution for four minutes, thus, these samples will be named "AS" which stands for alkaline solution. Then, the sample was dried one more time with tissue paper.

Time evolution of $\mathrm{I}_{\mathrm{DS}}$ current up to $600 \mathrm{~s}$ was also investigated. Scanning Electron Microscopy (SEM) top view and cross section images were used to investigate the morphology of the samples.
AUTOLAB $^{\circledR}\left(\right.$ PGSTAT302N, METROHM $\left.^{\circledR}\right)$ was used to perform both Cyclic Voltammetry (CV) and Electrochemical Impedance Spectroscopy (EIS). Such analyses were done by varying the $\mathrm{pH}$ of a bulk solution between the values 2 to 12 . The acidic solutions were done with titration of mili Q water using Sulfuric Acid $\left(\mathrm{H}_{2} \mathrm{SO}_{4}\right)$, and the alkaline solutions were done titrating mili $\mathrm{Q}$ water using Sodium Hydroxide $(\mathrm{NaOH})$. The configuration used for both analyses was based on three electrodes: a reference electrode (Silver/ Silver Chloride), a working electrode (FTO sample) and a counter electrode (Platinum net) ${ }^{42,43}$. In such configuration, electrical current is flowing between the counter electrode and working electrode while the voltage is being monitored between reference electrode and working electrode. The main advantage performing these measurements through three different electrodes is to avoid any fluctuation in collected data. The cyclic voltammetry was done at a rate of $50 \mathrm{mV} / \mathrm{s}$ in 3 cycles. The voltage variation was taken around the potential of $0 \mathrm{~V}$ and was varied in two different potential windows: from $-0.5 \mathrm{~V}$ to $0.5 \mathrm{~V}$ and $-1.0 \mathrm{~V}$ to $1.0 \mathrm{~V}$.

EIS is a very powerful technique to describe electrode/ electrolyte electrochemical interfaces on steady state. Through this technique it is possible to build a resistor-capacitor (RC) equivalent circuit. The three electrode configuration was similarly used ${ }^{44,45}$. The applied AC signal presented a vast frequency spectrum from $10^{-2} \sim 10^{6} \mathrm{~Hz}$, without any dc bias. The technique was used to establish a better understanding of the kinetic mechanisms involved on the sorption of ions by the FTO.

\section{Results and Discussion}

\subsection{Cleaning process: initial and in between re- usage}

$\mathrm{V}_{\mathrm{DS}}$ and $\mathrm{V}_{\mathrm{GS}}$ data measurements using sample $\mathrm{S} 21$ are presented in Figure 2 as proof of concept of the influence of cleaning process on the final results. Figure 2(a) corresponds to the $\mathrm{V}_{\mathrm{DS}}$ mode, where solid circles represent the data from a sample cleaned with alkaline solution (labeled as AS), and open squares represent the data from the sample cleaned with water only (labeled as Wt). The solid lines correspond to linear fittings. CR stands for crescent mode, i.e. increasing $\mathrm{pH}$ value. The largest current values are always observed for the AS mode for each $\mathrm{pH}$ value. The difference between both cleaning processes is very clear.

As cleaning process, alkaline solution seems to provide a more effective and deeper cleaning of the surface than just water. The hydroxyl groups, abundant in alkaline solutions, react with $\mathrm{H}^{+}$protons previously adsorbed on the surface of the sample, thus forming water. This neutralizes the sample providing a better chemical cleaning. A cleaner surface allows a larger number of ions to be adsorbed on the next measurement. When the sample is cleaned with 


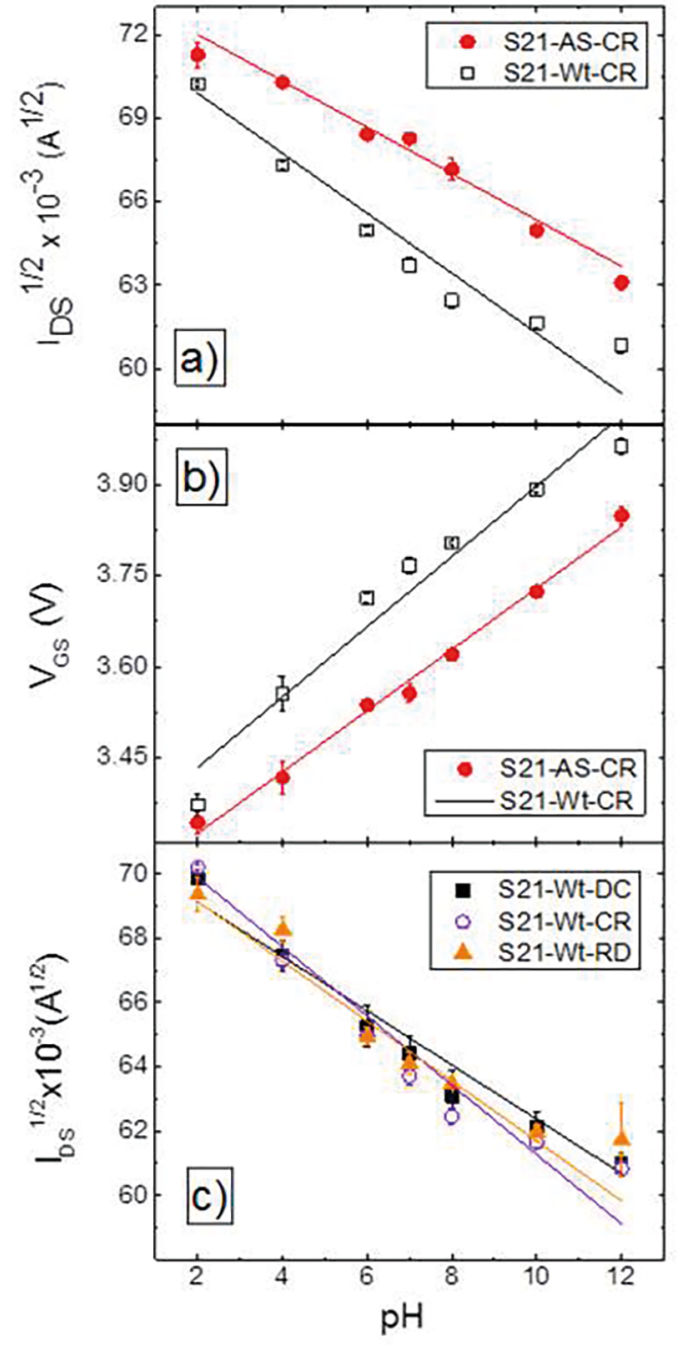

Figure 2. Response for sample S21 for the two different cleaning processes. a) Typical VDS mode response for AS (slope of the line $-0,00083 \pm 0,00007 \mathrm{~A}^{1 / 2} / \mathrm{pH}$ ) and Wt (slope of the line $-0,00108 \pm 0,00009$ $\mathrm{A}^{1 / 2} / \mathrm{pH}$ )cleaning processes. b) Typical VGS mode response for AS (slope of the line $0,0505 \pm 0,002 \mathrm{mV} / \mathrm{pH}$ ) and $\mathrm{Wt}$ (slope of the line $0,058 \pm 0,006 \mathrm{mV} / \mathrm{pH}$ ) cleaning processes. $\mathrm{pH}$ crescent configuration. c) $\mathrm{pH}$ measurement sequence influence on sensor's response. Symbols: square $\rightarrow$ decrescent (slope of the line $-0,00084 \pm 0,00005 \mathrm{~A}^{1 / 2} / \mathrm{pH}$ ); triangle $\rightarrow$ random (slope of the line $-0,00093 \pm 0,00009 \mathrm{~A}^{1 / 2} / \mathrm{pH}$ ); circle $\rightarrow$ crescent (slope of the line $-0,00108 \pm 0,00005 \mathrm{~A}^{1 / 2} / \mathrm{pH}$ ).

water only, some residual ions may remain adsorbed on its surface. These ions are enough to shield the sample against a larger attachment of new ions on the next measurement, and consequently the measured signal is smaller.

Figure 2(b) shows the results for sample S21 for $\mathrm{V}_{\mathrm{GS}}$ measurement mode. The solid circles represent the AS cleaning process and the open squares represent $\mathbf{W t}$ cleaning process. The sensitivity of the samples is extracted from a linear fitting of these data. Note that for the $\mathbf{W t}$ cleaning process this value was $58.0 \pm 6.0 \mathrm{mV} / \mathrm{pH}$, while the corresponding value for the AS cleaning was $50.5 \pm 2.0$
$\mathrm{mV} / \mathrm{pH}$. The observed difference of about $13 \%$ could lead to misleading conclusions in real usage of the device. The highest sensitivity allowed by Nernst equation and accepted by the scientific community is $59 \mathrm{mV} / \mathrm{pH}^{46}$.

This section leads to the conclusion that the cleaning process can be crucial for the sensor's behavior in the future. Cleaning the sample with alkaline solution can increase its current response. However, this cleaning process can decrease the sensitivity of the sensor. On the other hand, cleaning the sample with water can decrease the current response of the sensor, but improves its sensitivity. Thus in the use of any EGFET sensor it is very important that the cleaning process is clearly specified, once the same sample can present varied final sensitivities.

\subsection{Measurements with increasing and decreasing $\mathrm{pH}$ sequences}

The chosen sequence of $\mathrm{pH}$ values for subsequent measurements might also be important. According to the specific material used in the sensing film, eventual memory effects could also jeopardize a final result of the sensors. Three different sequences were chosen for this work: random (from now on also named RD), crescent (from now on also named CR) and decrescent (from now on also named DC). For the crescent sequence the measurements were performed on solutions with pHs ranging constantly increasing from 2 to 12 . The inverse was used for the decrescent sequence where $\mathrm{pHs}$ values of solutions were constantly decreasing from 12 to 2 . For the random sequence the $\mathrm{pH}$ values were $12,4,7,2,10,6$ and 8 .

Figure 2 (c) shows the results for sample S21, using the three sequences describe above. Only water cleaning process was used between measurements. The solid lines correspond to linear fittings. There is a very slight difference between signals' amplitudes and linear regression slopes for different measurement sequences. The linear regressions could suggest, at most, that the decrescent sequence leads to larger data values than the other sequences at high $\mathrm{pHs}$. This could happen due to the gradually increasing amount of $\mathrm{H}^{+}$protons in contact with the surface, as the $\mathrm{pH}$ decreases. Nevertheless, as observed in Figure 2 (c), the effect in the present measurements is weak. For the crescent sequence, the first measurement at $\mathrm{pH} 2$ presented the largest amplitude. The resulting fitted curve for the whole sequence presented the largest slope. At the very first measurement, the surface of the sample gets in contact with the largest possible concentration of $\mathrm{H}^{+}$protons. The surface adsorbs a great number of ions within its mesopores (spaces between the polycrystalline grains of the sample). The water cleaning process is not totally effective and some ions still remain adsorbed within the surface even after the cleaning process. These remaining ions somehow shield the surface against the adsorption of new ions for experiments using other $\mathrm{pH}$ values. The smaller response for other $\mathrm{pHs}$ leads to the larger slope. 
For the random sequence all the effects are averaged out, so its slope lies in the middle. Reusable sensors situation most likely approaches the random sequence presented above. In summary, in any investigation, the proper presentation of the used sequence is a key issue.

\subsection{Electrical resistivity of the FTO sensing film}

Samples with electrical sheet resistance in the range of $10 \Omega / \mathrm{sq}$ and $100 \Omega / \mathrm{sq}$ were used to investigate the influence of electrical properties of sensing films on the final response of the sensor. The cleaning process used water only as described before. Figure 3(a) presents the results for crescent $\mathrm{pH}$ sequence, while Figure 3(b) presents the results for decrescent $\mathrm{pH}$ sequence. Besides the fact that the curves seem very close accordingly to the standard deviations, some discussions might still be conducted.

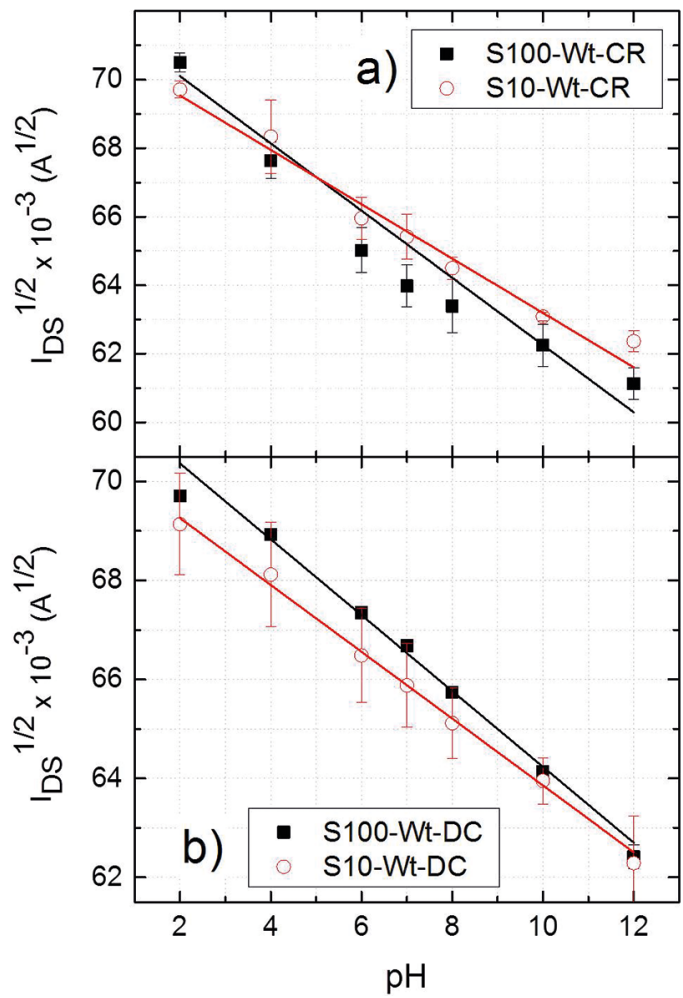

Figure 3. Resistivity influence for samples of $10 \Omega / \mathrm{sq}$ (red circles) and $100 \Omega / \mathrm{sq}$ (black squares) cleaned with water, on $\mathrm{V}_{\mathrm{DS}}$ configuration for two $\mathrm{pH}$ sequences: a) Crescent sequence; b) Decrescent sequence.

As expected from the previous discussion about the crescent pH sequence (Figure 2 (c)), samples in this configuration are harder to be properly cleaned with water. The sequence starts with the highest $\mathrm{H}^{+}$protons concentration. The water usage does not clean the sample's surface with perfection and part of the ions remains adsorbed within the mesopores after the cleaning process. According to Figure 3(a) the results for sample S10 present a smaller slope, with current values slightly above the ones for sample S100. This might be explained by how fast the samples are cleaned, once all the samples were cleaned the same way and by the same time. It seems that the less resistive sample S10 has a faster cleaning process than the more resistive sample S100. This means that the ion adsorption and desorption processes for sample S10 are faster than for sample S100. This would explain why just the first point at $\mathrm{pH} 2$ for sample $\mathrm{S} 100$ had larger amplitude than for sample S10. The first measured point already adsorbs a great number of $\mathrm{H}^{+}$protons within the surface of the sample.

If sample S100 is slower cleaned than S10 and both of them were cleaned during the same amount of time, consequently sample S100 will present more residual ions in its surface. These ions will shield the surface against adsorption of new ions in the next measurement ( $\mathrm{pH} 4)$, and the resulting measured current will be smaller than for $\mathrm{S} 10$. The sensor's sensitivity are $\mathrm{S} 100(\mathrm{Wt}, \mathrm{CR})=53 \pm 5 \mathrm{mV} / \mathrm{pH}$ and $\mathrm{S} 10(\mathrm{Wt}, \mathrm{CR})=46 \pm 5 \mathrm{mV} / \mathrm{pH}$.

Figure 3(b) shows the results for the decrescent $\mathrm{pH}$ sequence for the same samples. As previously discussed, the decrescent $\mathrm{pH}$ sequence benefits a more organized adsorption of ions once this sequence gradually increases the concentration of $\mathrm{H}^{+}$protons in each new measurement. This sequence favors the water cleaning process by the same reason. As observed, sample S100 presents the larger amplitudes, just because it was better cleaned. However, the difference between the current values for both samples seems to increase for decreasing $\mathrm{pH}$ values. Sample S100 presents, once again, the largest slope. The lower the $\mathrm{pH}$ value, the larger the concentration of $\mathrm{H}^{+}$protons and more pronounced would be the differences due to the cleaning process. The sensitivities were $\mathrm{S} 100(\mathrm{Wt}, \mathrm{DC})=62 \pm 4 \mathrm{mV} /$ $\mathrm{pH}$ and $\mathrm{S} 10(\mathrm{Wt}, \mathrm{DC})=46 \pm 1 \mathrm{mV} / \mathrm{pH}$.

Because the cleaning process was kept constant for all measurements in these experiments, the sensors characteristics and measurement sequence became more influent on the sensors response. As shown in Figure 3(a), at a crescent measurement sample S100 is not capable to be completely cleaned by the cleaning process: the response for the next $\mathrm{pH}$ values is affected by a memory effect. The following measurements have smaller amplitude than the correspondent measurements for sample S10. Thus, sample S100 is slower cleaned than sample S10. This hypothesis is confirmed by Figure 3(b), where sample S100 was benefited by the measurement sequence, now crescent on concentration of $\mathrm{H}^{+}$ions (decrescent $\mathrm{pH}$ ). This sequence slowly increases the concentration of $\mathrm{H}^{+}$ions allowing sample $\mathrm{S} 100$ to efficiently adsorb a bigger number of ions as their concentration increases. The adsorption process is faster for sample S10, so smaller changes are observed.

The time evolution of the response of the sensors can present additional information about how fast a sample can achieve an equilibrium condition. For this experiment, 
both $\mathrm{V}_{\text {Ref }}$ and $\mathrm{V}_{\mathrm{DS}}$ were kept constant at $5 \mathrm{~V}$ and the $\mathrm{I}_{\mathrm{DS}}$ time evolution was monitored for $600 \mathrm{~s}$. Two buffer solutions were used: one acid at pH 2 and another alkaline at $\mathrm{pH} 8$. The results of such experiments are presented in Figure 4, and they confirm the different ion exchange velocities for both samples. Figure 4(a) shows that for $\mathrm{pH} 2$, sample S10 presents a fast decreasing current process that lasts for about 100s. Sample S100 does not present the same behavior and equilibrium was achieved only after $500 \mathrm{~s}$. The same qualitative behavior is observed in Figure 4(b) for a larger $\mathrm{pH}$ equal to 8 . In this case, the first decay process for sample $\mathrm{S} 10$ seems to end in one third of the previous time in Figure 4(a). On the other hand, sample S100 did not reach equilibrium even after 600s. This shows that the more resistive sample S100 achieves equilibrium much slower than sample S10, regardless of the electrolyte solution, acid or alkaline.

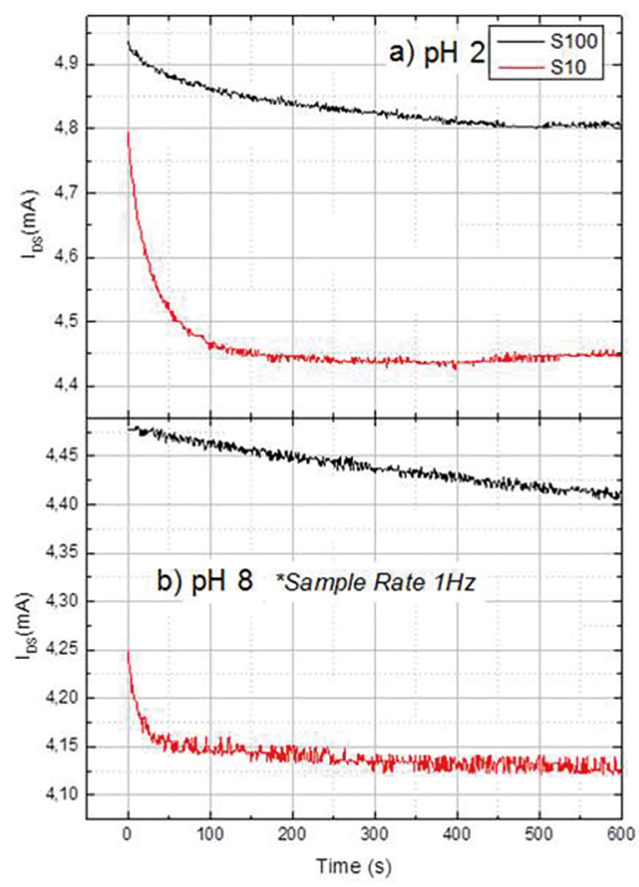

Figure 4. Current $\mathrm{I}_{\mathrm{DS}}$ variation in time for samples $\mathrm{S} 10$ and $\mathrm{S} 100$ when submerged into two different solutions: a) Acid solution ( $\mathrm{pH}$ 2); b) Alkaline solutions ( $\mathrm{pH} 8$ ).

The FTO samples are constituted by grains and consequently mesopores as shown by the SEM pictures in Figure 5. The data for sample S10 are presented at the left-side pictures a) trough c). Figure 5(a) shows the SEM top view of an original S10 sample. The cross section of an original sample is presented in Figure 5(b). Sample S10 is $670 \mathrm{~nm}$ thick. The cross-section of a non-working sample after several re-usages (hereon called dead sample) is presented in Figure 5(c). The corresponding information for sample S100 are presented at the right side pictures d) through g) in Figure 5. Note that sample S100 is $206 \mathrm{~nm}$ thick only.

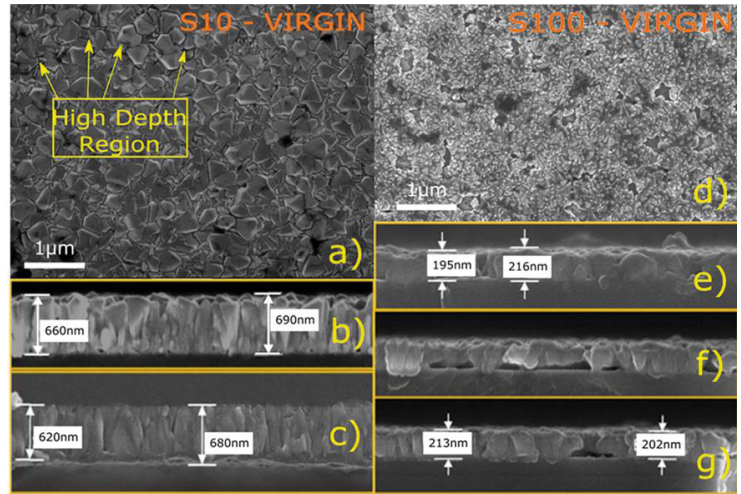

Figure 5. Scanning Electronic Microscopy. a) Top View of the surface of sample S10 with presence of High Depth Region or Mesopores; b) Lateral view of a virgin sample S10; c) Lateral view of a dead sample S10; d) Top View of the surface of sample S100; e) Lateral view of a virgin sample S100; f) Lateral view of a dead sample S100 with presence of Mesopores created by the detachment of sample from the glass; g) Lateral view of a dead sample S100 with presence of Mesopores created by the detachment of sample from the glass.

The micro grains are clearly seen in Figure 5(a). An average grain size of $200 \mathrm{~nm}$ was measured. The union of two consecutive grains is not totally sealed. The comparison of Figure 5(a) and (d) suggest that sample S10 has a more homogeneous surface. The heterogeneity of sample S100 is also notoriously confirmed by the presence of several holes on its surface. This physical characteristic of both samples helps to corroborate the idea that for sample S100 it is harder to remove the adsorbed ion. This makes the proper cleaning a very slow process.

The lateral views shown in Figures 5(b) and (e) for the virgin S10 and S100 samples reveal a columnar structure. Some empty spaces under the surface are also observed. The presence of mesopore tunnels might be inferred. Those pores increase the effective surface area of the samples and eventually can allow a larger ion adsorption. These pores would also make it more difficult to reset the samples after the first use.

The cross section in Figure 5(c) suggests that the dead S10 sample was seriously changed. There are much more wrinkles on this sample than before. This difference makes the cleaning process harder and consequently the sample loses its sensing properties. Figures 5(f) and (g) shows some more interesting effects for sample S100. Large holes under the entire film can be observed. Ion trapping must be more severe in this case, leading also to the death of the sample.

\subsection{Cyclic voltammetry and impedance spectroscopy}

Cyclic voltammetry and impedance spectroscopy were used as complementary experiments. Even though both techniques require a dynamic process with current flow, opposite to the previous EGFET measurements, the results will corroborate our previous findings and conclusions. 
An example of a cycle from the cyclic voltammetry experiment is shown in Figure 6, for sample S10 in a solution with $\mathrm{pH} 7$ without application of any offset voltage. One cycle was divided in two different regions defined by the potential applied: positive potential (red area in Figure 6) and negative potential (green area in Figure 6). The areas represent the total accumulated charge. The most interesting characteristic of the sample provided by this graph is the equality between the number of charges accumulated in the sample for both negative and positive potential (green area is the same as red area). This means that the sample doesn't prioritize the adsorption of Hydrogen ions $\left(\mathrm{H}^{+}\right)$or Hydroxyl groups $\left(\mathrm{OH}^{-}\right)$on its surface.

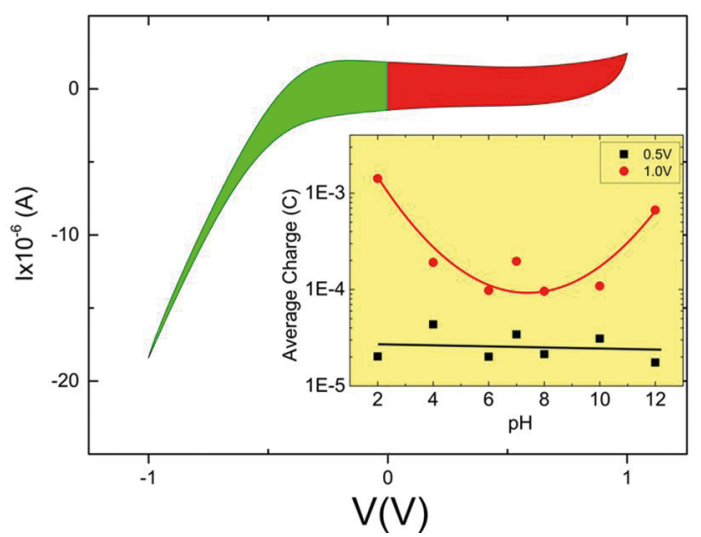

Figure 6. Typical cycle provided by cyclic voltammetry for a FTO sample. For this graphic a sample of $10 \Omega /$ sq was used. No offset voltage was applied to the sample. Inset) Results from the cyclic voltammetry experiments. Average of charges accumulated at the sample' surface for different $\mathrm{pHs}$ and different voltages applied to the film. The maximum error for $0.5 \mathrm{~V}$ is $\pm 3.9 \mathrm{E}-5 \mathrm{C}$ and for $1.0 \mathrm{~V} \pm 1.7 \mathrm{E}-3 \mathrm{C}$

The inset of Figure 6 shows the average charge accumulated at the surface of sample S10 for two different cycles as a function of $\mathrm{pH}$. This charge is equal to the area inside the curves. Positive charges are accumulated in the surface for pHs between 2 and 7. Negative charges are accumulated in the surface for pHs between 7 and 12. For the first cycle, red circles represent the charges accumulated for $1 \mathrm{~V}$ voltammetry. For the second cycle, black squares represent the charges accumulated for $0.5 \mathrm{~V}$ voltammetry. The lines in inset of Figure 6 are just guide to the eyes, not representing any mathematical analyses or tool.

Charges calculated for $0.5 \mathrm{~V}$ potential presents an almost flat distribution. Certainly, the corresponding electric field is not strong enough to move a significant amount of ions, so the effect doesn't become clear. Charges calculated for $1 \mathrm{~V}$ potential presents a U-shaped distribution. The effect can be explained by the affinity of the sample with both $\mathrm{H}^{+}$or $\mathrm{OH}^{-}$, with a slightly higher affinity for $\mathrm{H}^{+}$. This ion is extremely tiny and can move around the solution and through the sample with relatively higher freedom when compared to Hydroxyl groups. This easiness of $\mathrm{H}^{+}$ion movement allows it to reach the sample in greater number than Hydroxyl groups, thus generating a larger signal.

Typical Nernstian sensitivities of EGFET $\mathrm{pH}$ sensors lies always bellow $60 \mathrm{mV} / \mathrm{pH}$. The total $\Delta \mathrm{V}$ value expected for an EGFET experiment varying the $\mathrm{pH}$ scale from 2 to 12 would then be lower than $1 \mathrm{~V}$. A better potential for cyclic voltammetry to address an analysis to complement EGFET data should be, certainly, bellow $1 \mathrm{~V}$. The potential of $0.5 \mathrm{~V}$ is close enough to real potentials applied between the extremes of a pH scale during EGFET measurements, so, this is the only one voltage used in the test. It is a characteristic of the MOSFET (not shown in this work): the necessary potential $\mathrm{V}_{\mathrm{GS}}$ directly applied to the MOSFET to achieve the same current amplitude $\mathrm{I}_{\mathrm{DS}}$ for $\mathrm{pH} 2$ is about $0.5 \mathrm{~V}$ above the used $\mathrm{V}_{\text {Ref }}$ In a similar behavior, the necessary potential $\mathrm{V}_{\mathrm{GS}}$ directly applied to the MOSFET to achieve the same current amplitude $\mathrm{I}_{\mathrm{DS}}$ for $\mathrm{pH} 12$ is about $0.5 \mathrm{~V}$ below the used $\mathrm{V}_{\text {Ref }}$ In conclusion, the closest behavior of charge accumulation on the FTO film can be found on the squares of inset of Figure 6.

A typical FTO response for impedance spectroscopy experiments is shown in Figure 7(a) in a Nyquist diagram. The experiment was accomplished using sample S10. The other samples should show a similar behavior for this test since this is a test to study a general behavior between the surface of the sample and the electrolyte solution. In such graph, Z' represents the real part of the total impedance while Z" represents the imaginary part of total impedance. So, the real impedance is related to the size of semi-circle formed by the lines in Figure 7(a) in Z' axis (where the lines touch $Z$ ' axis). The resistive part of the impedance increases for decreasing $\mathrm{H}^{+}$concentration on acidic solutions (going from $\mathrm{pH} 2$ to 6) or for decreasing $\mathrm{OH}^{-}$concentration on alkaline solutions (going from $\mathrm{pH} 12$ to 8 ). This behavior is directly related with the size of the first semi-circle of each curve and the effect is better seen in the inset, for low Z' values in Figure 7(a). For pHs 2 and 12, where $\mathrm{H}^{+}$ and $\mathrm{OH}^{-}$have their highest concentrations, respectively, the first semi-circle is not observed. This characteristic is directly related to the high capacitance and low resistivity of the sample on such environments. This assumption is corroborated by the graph in Figure 7(b). For example, at $1 \mathrm{kHz}$, most of the capacitances lye in the $100 \mathrm{pF}$ to $1 \mathrm{nF}$ range. The exceptions are the capacitances for solutions at pHs 2 and 12, once they are at least 3 orders of magnitude larger. The simplest possible equivalent circuit for the system is presented at the bottommost part of Figure 8. $R_{S}$ represents the resistance of the solution, $R_{D L}$ the resistance of the double layer and $\mathrm{C}_{\mathrm{DL}}$ the capacitance of the double layer. 


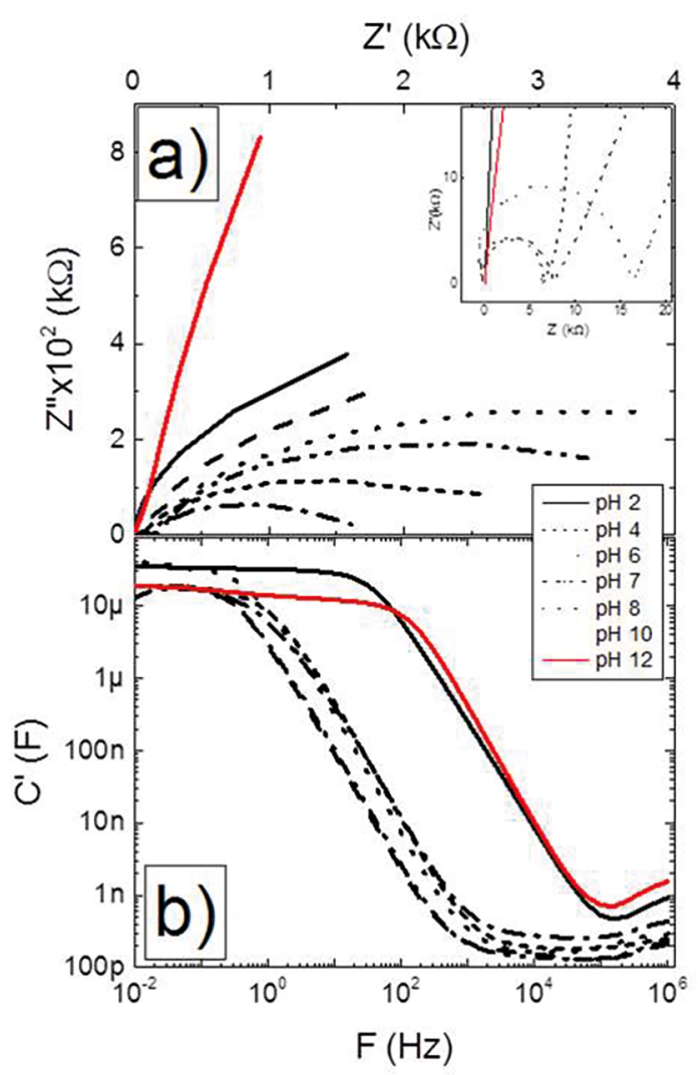

Figure 7. a) Spectroscopy impedance results. Nyquist diagram for FTO sample in different pHs $(2,4,6,7,8,10$ an 12). b) Capacitance Diagram for a typical FTO sample.

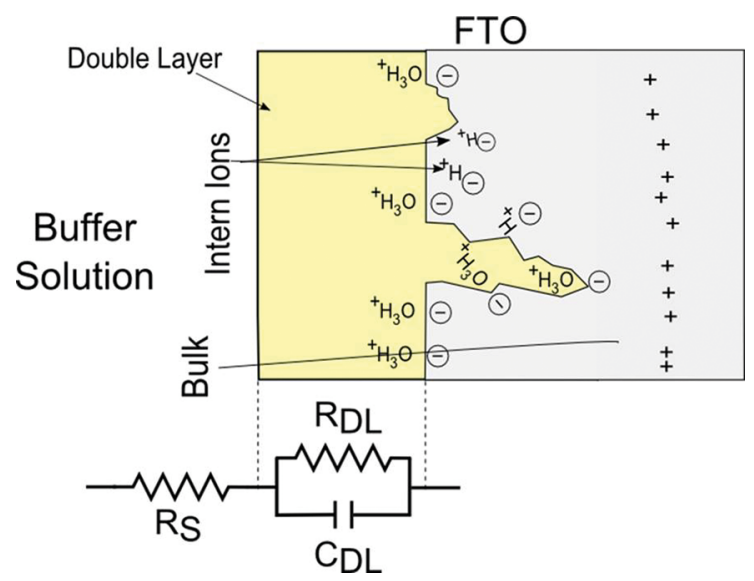

Figure 8. Schematic of charges distribution all over the FTO sample at the top. Equivalent electric circuit at the bottom.

\section{Physical-Chemical Model}

Figure 8 resumes the most important and relevant effects related to the ion adsorption mechanisms. Other more detailed studies about metal-oxide interfaces and their electrostatic potentials were already reported elsewhere ${ }^{47}$. Nevertheless, our intention is to describe a simpler model describing our findings. According to what was previously presented, there are basically three main regions where an ion can be adsorbed in a sample:

i) The double-layer at the surface. This is a layer that extends all over the surface of a sample, right in front of it. This is the main region where ions are adsorbed and solvated ${ }^{48}$. When hydrogen ions are adsorbed on this layer, a hydronium $\left(\mathrm{H}_{3} \mathrm{O}^{+}\right)$ molecule is formed, as shown at the left part of Figure 8. A water molecule naturally present in the double-layer is attacked by the $\mathrm{OH}^{-}$group which can pull out a hydrogen atom from $\mathrm{it}^{49}$. A hydroxyl group is left behind on that place ${ }^{44}$ (not shown in Figure 8), adsorbed within the double layer. As a consequence of ion adsorption in the double layer, a counter-ions layer is formed just behind the surface, inside the sample. Figure 8 shows the attachment of hydrogen ions in the double-layer. The counter-ions are illustrated as anions (negative charge). Once the film is naturally isoelectric, the negative counter-ions that appear right behind the surface of the sample need to travel from the bulk. In this case, the bulk will present an accumulation of positive charges, as shown in the rightmost part of Figure 8.

ii) The internal surfaces due to structural imperfections. The surface imperfections (due to the film production) can even extend a certain range towards the substrate as previously discussed and illustrated in Figure 8. Some of these defects were already reported elsewhere ${ }^{48}$. Some few hydrogen ions are assumed to reach these internal surfaces, thus becoming intern ions. They are indicated in the middle part of Figure 8 . These ions cannot penetrate deeply inside the film and stop right on the internal surfaces. They are neutralized by their proper counter-ions which make the bulk charge to become more positive on this specific case.

iii) The bulk itself. Some ions from solution might also diffuse towards the bulk.

Each one of these regions can be modeled as a virtual capacitor, respectively $\mathrm{C}_{\mathrm{DL}}$ for double-layer, $\mathrm{C}_{\mathrm{II}}$ for the internal ions, and $\mathrm{C}_{\mathrm{B}}$ for the bulk. They can be imagined in a series configuration and their capacitance can be added to each other in order to form the final equivalent capacitor:

$$
\frac{1}{C_{e q}}=\frac{1}{C_{D L}}+\frac{1}{C_{I I}}+\frac{1}{C_{B}}
$$

However, the FTO thin film is a highly conductive semiconductor material and the two capacitors associated to the intern ions and to the ions in the bulk will play a very small role. The bulk low resistance will basically short-circuit the capacitors and the system will in fact be fairly described by a RC model. With this simplification, the equivalent capacitance is just related to the double layer capacitor, as presented in the equivalent circuit at the bottommost part of Figure 8 . 
According to the definition of capacitance, once there is no surface and structural variation and given the electric potential difference is known, the amount of net charge can be inferred. Any variation on the potential applied to the surface of the sample will also result on a variation of accumulated charge. This leads to construction of Figure 9. The lines found in Figure 9 are just guides to the eyes. They do not represent any mathematical function or analysis tool.

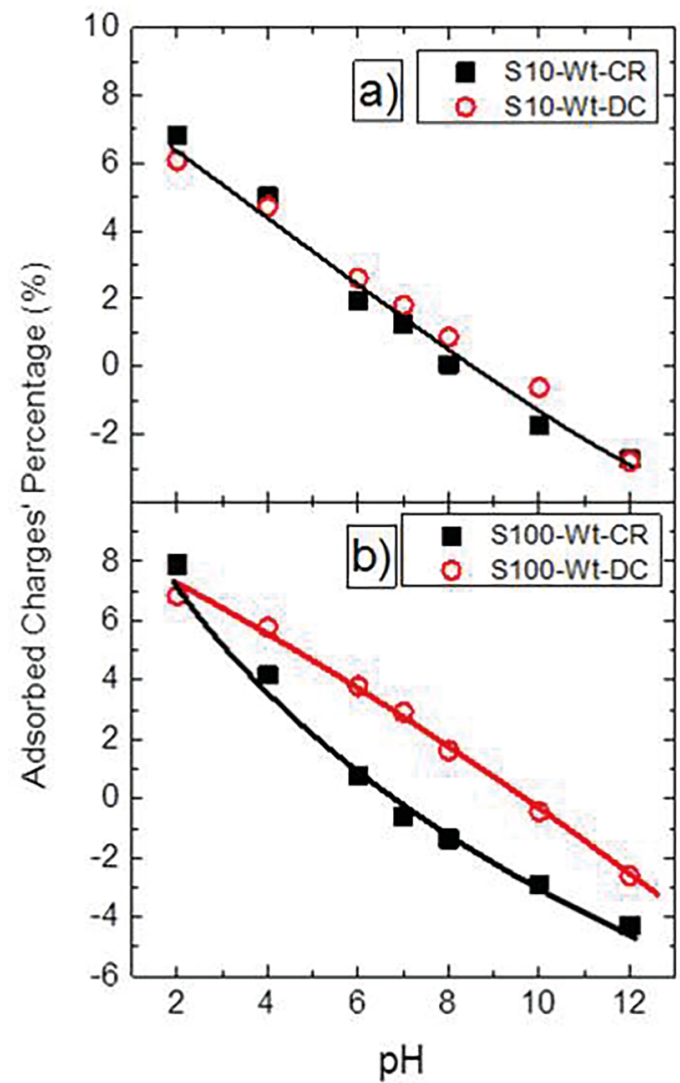

Figure 9. Percentage charge's variation for samples cleaned in water: a) Crescent and Decrescent $\mathrm{pH}$ sequence for Sample S10; b) Crescent and Decrescent $\mathrm{pH}$ sequence for Sample S100. The lines shown in the figure are just eye guides. They do not represent any kind of mathematical instrument or analysis.

Figure 9(a) shows the percentage of charge variation for the case of sample S10. The data for the two different sequences of measurement, crescent and decrescent, are plotted in this figure. Once this sample S10 is cleaned faster, there is almost no difference between the two $\mathrm{pH}$ sequences. So, the variation of charge is basically the same for both sequences when the sample is cleaned in water.

Figure 9(b) shows the charge variation for sample S100 on both $\mathrm{pH}$ sequences, when it was also cleaned in water. Now, each measurement sequence has a specific behavior. As discussed, and confirmed by Figure 9(b), the best $\mathrm{pH}$ sequence possible when this sample is cleaned in water is the decrescent one, where $\mathrm{H}^{+}$protons are gradually introduced to the surface. So, this sequence can be used as a reference for comparison purposes, and the slow response of sample S100 leads to the charge difference reduction for the smallest $\mathrm{pH}$. This finding corroborates our previous analyses done with EGFET system.

\section{Conclusions}

It was shown that the cleaning process can influence the final response of FTO films when used as an EGFET $\mathrm{pH}$ sensor. Different response characteristics are prioritized according to the cleaning procedure and solution. Alkaline solution raises the sensor's response amplitude, but lowers its sensitivity. On the other hand, cleaning the sample in water lowers the sensor's response amplitude and raises sensor's sensibility. The FTO film electrical resistivity and morphology were shown to play an important role on the cleaning process. Less resistive samples have a relatively homogeneous surface. Thus, through a fast and simple cleaning process they can return fairly close to their original state. More resistive samples, on the other hand, need a slower cleaning process. This kind of sample has a very heterogeneous surface that is hard to be cleaned. A physical-chemistry mechanism for charges adsorption on the surface of the sample was proposed. The effective capacitance was analyzed at different $\mathrm{pHs}$. As shown, less resistive samples show a small difference on the amount of adsorbed charges as a function of $\mathrm{pH}$ measurement sequence. Bigger differences were found for the most resistive samples according to the crescent or decrescent $\mathrm{pH}$ sequence. That occurs because the crescent $\mathrm{pH}$ sequence disturbs the sample's response. This work showed that fundamental aspects, physical characteristics and experimental setup configuration have direct influence on FET biosensor's response.

\section{Acknowledgements}

This work was supported by FAPESP(2014/09562-4), CAPES and CNPq (302269/2012-6) Brazilian agencies.

\section{References}

1. Chiu YS, Tseng CY, Lee CT. Nanostructured EGFET pH Sensors With Surface-Passivated ZnO Thin-Film and Nanorod Array. IEEE Sensors Journal. 2012;12(5):930-934.

2. Yao PC, Lee MC, Chiang JL. Annealing Effect of Sol-Gel $\mathrm{TiO}_{2}$ Thin Film on pH-EGFET Sensor. In: 2014 International Symposium on Computer, Consumer and Control; 2014 Jun 10-12; Taichung, Taiwan. IEEE. p. 577-580.

3. Batista PD, Mulato M. Polycrystalline fluorine-doped tin oxide as sensoring thin film in EGFET $\mathrm{pH}$ sensor. Journal of Materials Science. 2010;45(20):5478-5481. 
4. Koktysh DS, Liang X, Yun BG, Pastoriza-Santos I, Matts RL, Giersig M, et al. Biomaterials by Design: Layer-By-Layer Assembled Ion-Selective and Biocompatible Films of $\mathrm{TiO}_{2}$ Nanoshells for Neurochemical Monitoring. Advanced Functional Materials. 2002;12(4):255-265.

5. Li Z, Yang R, Yu M, Bai F, Li C, Wang ZL. Cellular Level Biocompatibility and Biosafety of $\mathrm{ZnO}$ Nanowires. Journal of Physical Chemistry C. 2008;112(51):20114-20117.

6. Wu R, Chen X, Hu J. Synthesis, characterization, and biosensing application of $\mathrm{ZnO} / \mathrm{SnO}_{2}$ heterostructured nanomaterials. Journal of Solid State Electrochemistry. 2012;16(5):1975-1982.

7. He R, Zhao L, Liu Y, Zhang N, Cheng B, He Z, et al. Biocompatible $\mathrm{TiO}_{2}$ nanoparticle-based cell immunoassay for circulating tumor cells capture and identification from cancer patients. Biomedical Microdevices. 2013;15(4):617-626.

8. Jang JM, Park SJ, Choi G, Kwon TY, Kim KH. Chemical state and ultra-fine structure analysis of biocompatible $\mathrm{TiO}_{2}$ nanotube-type oxide film formed on titanium substrate. Metals and Materials International. 2008;14:457.

9. Cao Z, Sun LX, Zhou T, Luo YF, Zeng JL, Long S, et al. Investigation on Electronic Signals for Detection of Target DNA Molecule Based on Extended Gate FET Sensing Chip. Advanced Materials Research. 2011;236-238:1923-1926.

10. Chen CP, Ganguly A, Lu CY, Chen TY, Kuo CC, Chen RS, et al. Ultrasensitive in Situ Label-Free DNA Detection Using a Gan Nanowire-Based Extended-Gate Field-Effect-Transistor Sensor. Analytical Chemistry. 2011;83(6):1938-1943.

11. Hsieh CH, Chen PH, Chen RH, Huang IY. Development of EGFET-based microsensors with high-sensitivity and highlinearity for dissolved oxygen and carbon dioxide detection. In: 2013 Transducers \& Eurosensors XXVII: The $17^{\text {th }}$ International Conference on Solid-State Sensors, Actuators and Microsystems; 2013 Jun 16-20; Barcelona, Spain. IEE. p. 2051-2054.

12. Yin LT, Lin YT, Leu YC, Hu CY. Enzyme immobilization on nitrocellulose film for $\mathrm{pH}$-EGFET type biosensors. Sensors and Actuators B: Chemical. 2010;148(1):207-213.

13. Chen JC, Chou JC, Sun TP, Hsiung SK. Portable urea biosensor based on the extended-gate field effect transistor. Sensors and Actuators B: Chemical. 2003;91(1-3):180-186.

14. Kim DS, Han DI, Park JE, Shin JK, Choi P, Lee JH, et al. S. An extended gate field effect transistor based protein sensor integrated with a Si micro-fluidic channel. In: The $13^{\text {th }}$ International Conference on Solid-State Sensors, Actuators and Microsystems, 2005. Digest of Technical Papers. TRANSDUCERS'05; 2005 Jun 5-9; Seoul, South Korea. IEEE, Volume 2. p. 2011-2014.

15. Chou JC, Lin CY. Sensing Properties and Stability Analysis of Miniaturized Dual-Mode Uric Acid Biosensor Based on $\mathrm{TiO}_{2}$ Extended Gate Field Effect Transistor. Sensor Letters. 2008;6(6):929-932.

16. Guan W, Reed MA. Extended Gate Field-Effect Transistor Biosensors for Point-Of-Care Testing of Uric Acid. Biosens. In: Prickril B, Rasooly A, eds. Biosensors and Biodetection Methods and Protocols, Volume 2: Electrochemical, Bioelectronic, Piezoelectric, Cellular and Molecular Biosensors. New York: Springer; 2017. p. 189-203.
17. Lee CT, Chiu YS, Ho SC, Lee YJ. Investigation of a Photoelectrochemical Passivated Zno-Based Glucose Biosensor. Sensors (Basel). 2011;11(5):4648-4655.

18. Batista PD. An embedded measurement system for the electrical characterization of EGFET as a $\mathrm{pH}$ sensor. Measurement Science and Technology. 2013;25(2):027001.

19. Li HH, Yang CE, Kei CC, Su CY, Dai WS, Tseng JK, et al. Coaxial-structured $\mathrm{ZnO} /$ silicon nanowires extended-gate fieldeffect transistor as pH sensor. Thin Solid Films. 2013;529:173176.

20. Huang BR, Lin TC. Leaf-like carbon nanotube/nickel composite membrane extended-gate field-effect transistors as $p \mathrm{H}$ sensor. Applied Physics Letters. 2011;99(2):023108.

21. Lee PY, Chang SP, Kuo PJ, Hsu EH, Chang SJ, Shei SC, et al. Sensing Performance of EGFET pH Sensors with CZTSe Nanoparticles Fabricated on Glass Substrates. International Journal of Electrochemical Science. 2013;8:3866-3875.

22. Batista PD, Mulato M, Graeff CO, Fernandez FJR, Marques FC. $\mathrm{SnO}_{2}$ extended gate field-effect transistor as $\mathrm{pH}$ sensor. Brazilian Journal of Physics. 2006;36(2a):478-481.

23. Batista PD, Mulato M. ZnO extended-gate field-effect transistors as $p \mathrm{H}$ sensors. Applied Physics Letters. 2005;87(14):143508.

24. Wang JL, Yang PY, Hsieh TY, Hwang CC, Juang MH. Ph-Sensing Characteristics of Hydrothermal Al-Doped ZnO Nanostructures. Journal of Nanomaterials. 2013;2013:152079.

25. Hsu JF, Huang BR, Huang CS, Chen HL. Silicon nanowires as pH sensor. Japanese Journal of Applied Physics. 2005;44(Pt 1 $4 \mathrm{~S}): 2626$.

26. Chi LL, Chou JC, Chung WY, Sun TP, Hsiung SK. Study on extended gate field effect transistor with tin oxide sensing membrane. Materials Chemistry and Physics. 2000;63(1):19-23.

27. Chin YL, Chou JC, Lei ZC, Sun TP, Chung WY, Hsiung SK. Titanium Nitride Membrane Application to Extended Gate Field Effect Transistor pH Sensor Using VLSI Technology. Japanese Journal of Applied Physics. 2001;40(Pt 1 11):6311.

28. Guan W, Duan X, Reed MA. Highly specific and sensitive non-enzymatic determination of uric acid in serum and urine by extended gate field effect transistor sensors. Biosensors and Bioelectronics. 2014;51:225-231.

29. Das A, Ko DH, Chen CH, Chang LB, Lai CS, Chu FC, et al. Highly sensitive palladium oxide thin film extended gate FETs as pH sensor. Sensors and Actuators B: Chemical. 2014;205:199205.

30. Campos RC, Cestarolli DT, Mulato M, Guerra EM. Comparative Sensibility Study of $\mathrm{WO}_{3}$ ph Sensor Using EGFET and Ciclic Voltammetry. Materials Research. 2015;18(1):15-19.

31. Yusof KA, Rahman RA, Zulkefle MAH, Herman SH, Abdullah WFH. pH sensitivity dependency on the annealing temperature of spin-coated titanium dioxide thin films. Jurnal Teknology. 2016;78(5-8):39-44.

32. Rahman RA, Zulkefle MAH, Yusof KA, Abdullah WFH, Mahmood MR, Herman SH. Characterization of $\mathrm{ZnO} / \mathrm{TiO}_{2}$ bilayer film for extended gate field-effect transistor (EGFET) based pH sensor. Jurnal Teknology. 2016;78(5-8):33-38. 
33. Sabah FA, Ahmed NM, Hassan Z. Almessiere MA. Influences of substrate type on the $\mathrm{pH}$ sensitivity of $\mathrm{CuS}$ thin films EGFET prepared by spray pyrolysis deposition. Materials Science in Semiconductor Processing. 2017;63:269-278.

34. Liu B, Hu R, Deng J. Studies on a potentiometric urea biosensor based on an ammonia electrode and urease, immobilized on a $\gamma$-aluminum oxide matrix. Analytica Chimica Acta. 1997;341(23):161-169.

35. Ali SMU, Nur O, Willander M, Danielsson B. A fast and sensitive potentiometric glucose microsensor based on glucose oxidase coated $\mathrm{ZnO}$ nanowires grown on a thin silver wire. Sensors and Actuators B: Chemical. 2010;145(2):869-874.

36. Afify HH, Momtaz RS, Badawy WA, Nasser SA. Some physical properties of fluorine-doped $\mathrm{SnO}_{2}$ films prepared by spray pyrolysis. Journal of Materials Science: Materials in Electronics. 1991;2(1):40-45.

37. Turgut G, Keskenler EF, Aydın S, Yılmaz M, Doğan S, Düzgün B. An investigation of the $\mathrm{Nb}$ doping effect on structural, morphological, electrical and optical properties of spray deposited F doped $\mathrm{SnO}_{2}$ films. Physica Scripta. 2013;87(3):035602.

38. Rakhshani AE, Makdisi Y, Ramazaniyan HA. Electronic and optical properties of fluorine-doped tin oxide films. Journal of Applied Physics. 1998;83(2):1049-1057.

39. Bergveld P. Thirty years of ISFETOLOGY: What happened in the past 30 years and what may happen in the next 30 years. Sensors and Actuators B: Chemical. 2003;88:1-20.

40. Nascimento RAS. Intra and extra-cellular glucose quantification by micro-nano-structured biosensors. [Thesis]. Ribeirão Preto: Faculdade de Filosofia, Ciências e Letras de Ribeirão Preto/ Universidade de São Paulo; 2015.
41. Chi LL, Chou JC, Chung WY, Sun TP, Hsiung SK. Study on extended gate field effect transistor with tin oxide sensing membrane. Materials Chemistry and Physics. 2000;63(1):19-23.

42. Sivakumar R, Gopalakrishnan R, Jayachandran M, Sanjeeviraja C. Investigation of X-ray photoelectron spectroscopic (XPS), cyclic voltammetric analyses of $\mathrm{WO}_{3}$ films and their electrochromic response in FTO/ $\mathrm{WO}_{3} /$ electrolyte/FTO cells. Smart Materials and Structures. 2006;15(3):877.

43. Kissinger PT, Heineman WR. Cyclic voltammetry. Journal of Chemical Education. 1983;60(9):702.

44. Bard AJ, Faulkner LR. Electrochemical Methods: Fundamentals and Applications. $2^{\text {nd }}$ ed. Hoboken: Wiley; 2000. 864 p.

45. Orazem ME, Tribollet B. Electrochemical Impedance Spectroscopy. $1^{\text {st }}$ ed. Hoboken: Wiley-Interscience; 2008.

46. Knopfmacher O, Tarasov A, Fu W, Wipf M, Niesen B, Calame $\mathrm{M}$, et al. Nernst Limit in dual-Gated Si-Nanowire FET Sensors. Nano Letters. 2010;10(6):2268-2274.

47. Streitz FH, Mintmire JW. Electrostatic potentials for metaloxide surfaces and interfaces. Physical Review B: Condensed Matter. 1994;50(16):11996-12003.

48. Brown GE Jr, Henrich VE, Casey WH, Clark DL, Eggleston C, Felmy A, et al. Metal Oxide Surfaces and Their Interactions with Aqueous Solutions and Microbial Organisms. Chemical Reviews. 1999;99(1):77-174.

49. Degen A, Kosec M. Effect of $\mathrm{pH}$ and impurities on the surface charge of zinc oxide in aqueous solution. Journal of the European Ceramic Society. 2000;20(6):667-673. 